\title{
Scaling of wind turbine aerodynamics: wind tunnel experiments
}

\author{
Michael Macháček ${ }^{1}$, Stanislav Pospíšil ${ }^{1, *}$ and Hrvoje Kozmar $^{2}$ \\ ${ }^{1}$ Czech Acad Sci, Inst Theoret \& Appl Mech, Prosecká 76, 19000 Prague, Czech Republic. \\ ${ }^{2}$ University of Zagreb, Faculty of Mechanical Engineering and Naval Architecture, Ivana Lučića 5, \\ 10000 Zagreb, Croatia.
}

\begin{abstract}
A small-scale wind turbine model was designed and manufactured to study its aerodynamic thrust force and the harvested flow energy. To provide a good understanding of the aerodynamics of the smallscale wind turbine at the low Reynolds number, the performance of three different types of blade airfoils was studied. The main motivation for the design of a new miniature wind turbine model was to achieve realistic values of the thrust force and the power coefficient on the model scale. A new blade profile with a thickness of $10 \%$ was designed and employed to reach the high tip-speed ratio, which is characteristic of contemporary wind turbines.
\end{abstract}

\section{Introduction}

Wind turbines represent nowadays complex engineering structures. They have been increasingly developed around the world due to a strong initiative on renewable energy production using the wind. During their lifetime, they are exposed to a harsh environment that may influence their lifetime performance and thus energy production. It is necessary to analyze those complex loads in detail and propose appropriate design solutions in order to increase their lifetime and energy production, possibly using the multi-disciplinary optimization methods, see e.g. [1].

While aerodynamic loads on wind turbines were previously thoroughly investigated for cases, where wind turbines were placed on flat terrain and open sea, the characteristics of atmospheric flow and turbulence around wind turbines in complex terrain, as well as their aerodynamic loads, are fairly unknown [2]. Nevertheless, studies on effects of complex terrain on wind-turbine wake characteristics, like for example [3] and [4] are quite rare, despite the fact that topographical features are likely to considerably influence the atmospheric turbulence and thus wind turbine aerodynamics. Due to the unsteady nature of the atmospheric boundary layer (ABL) flow, wind turbines are subject to different wind direction and magnitude, thus the on-site analysis is rather complicated and often impossible. For the experimental analysis of the rotating wind turbine in complex terrain, it is necessary to carefully select the size and design of the small-scale wind turbine and surrounding terrain models to satisfy the similarity criteria to the maximum possible extent.

*Stanislav Pospisil: pospisil@itam.cas.cz 
Previous studies [5] and [6] showed that the conventional airfoils made for the high Reynolds number regime have poor performance if they operate in the wind tunnel conditions, i.e. at low Reynolds numbers. In general, an optimum airfoil with the maximum lift to drag ratio at very low Reynolds numbers has been found to have a small thickness, sharp leading edge, see [7] and a 5\% circular arc camber, see [8].

Typically for the wind tunnel, downscaled wind turbines have a lower efficiency (performance) than their large-scale counterparts due to this factor together with the fact that the scaled turbines have to be small enough to ensure the so-called minimal blockage effect, see [9]. The present study describes a possible way to design a small-scaled wind turbine model characterized by relatively good efficiency along with a relatively agreement with the aerodynamic coefficients obtained using a theoretical approach.

\section{Wind turbine model}

The selected type of the wind turbine was Siemens Sapiens, which is a horizontal axis wind turbine equipped with three blades and the maximal power output of 6.0 MW. The fullscale diameter of its blades is $154 \mathrm{~m}$ and the rotor hub height is $115.5 \mathrm{~m}$ above the ground surface. The simulation length scale factor of the wind turbine model was 1:385. Thus, the diameter of the model wind turbine was $D=0.4 \mathrm{~m}$ and the model tower height was $H=0.3$ $\mathrm{m}$, as shown on Fig. $2 \mathrm{~b}$. The blockage of the wind tunnel test section was $3.8 \%$, so it was not necessary to perform blockage corrections. Achieving the low blockage in the wind tunnel test section is very important because the aerodynamics coefficients, as well as the power coefficient, are very sensitive to blockage effects.

Blades of the experimental wind turbine model were made from carbon fibre using a negative mould made by 3D printing technology. It was important to reach stiff and rigid blades, especially in the torsion mode because, in the experiments, the rotation speed was 5000 RPM, thus generating substantial aerodynamic and gyroscopic forces acting on the blades. The pitch angle of the blades was adjustable to enable setting various wind loading cases, see Fig. 2b. The aspect ratio of the blades was 12.5 , so the average length of the airfoil chord was approximately $15 \mathrm{~mm}$. The nacelle and hub models were made from plastic by $3 \mathrm{D}$ printing and the tower model was made from aluminium pipe with the $16 \mathrm{~mm}$ outer diameter.

\subsection{Design of the blades}

The blade design was based on the Glauert's optimum rotor criterion [10], which developed the blade element momentum theory based on the one-dimensional momentum theory as a simple method to predict the wind turbine or propeller performance. To define the twist angle of the entire length of the blades by Glauert, the only single nondimensional constant called the tip-speed ratio is required. This scale factor is defined as

$$
\lambda=\frac{\omega R}{U}
$$

where $U$ is the wind speed, $\omega$ is the angular velocity and $R$ is the radius of the wind turbine. For the experimental campaign, the ratio $\lambda=6$ was selected because in the real prototypes of wind turbines the tip-speed ratio is usually in the range 5 to 10 , depending on the wind speed. For the common type of large wind turbines characterized by a constant rotation speed, increasing the incoming freestream wind velocity causes a decrease of the tip-speed ratio. This ratio $\lambda=6$ is equal to high windy conditions as mentioned in [11]. 
a)

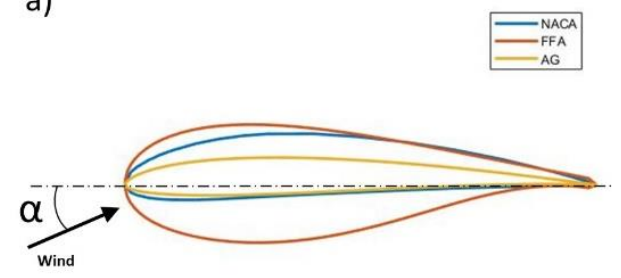

b)

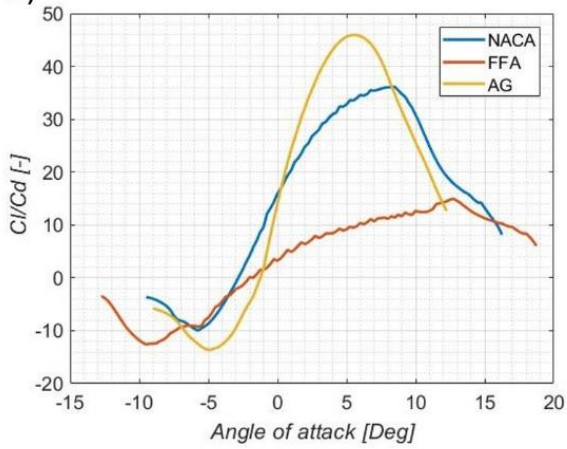

Fig. 1. The shape of three different airfoils (a) and their respective aerodynamic performance (b).

Experimental measurements were carried out using three types of airfoils. The first one was the FFA-W3-360 airfoil; it is typical for real wind turbines with their thickness equal to $30 \%$ of the chord length. The second type was well-known airfoil NACA 4412 with the thickness equal to $16 \%$ of the chord length. The third one was a special airfoil AG24, which is suitable for low Reynolds number conditions and it is characterized by the thickness equal to $10 \%$ of the chord length. The shape of the airfoils is shown in Fig. 1a. The model wind turbine was tested at the maximum Reynolds number $\operatorname{Re}=4 \cdot 10^{4}$, which is lower than Re for the respective prototype wind turbine, which is higher than $\operatorname{Re}=5 \cdot 10^{6}$.

Fig. 1b shows the ratio between the lift coefficient $C_{l}$ and the drag coefficient $C_{d}$ when changing the flow incidence angle. This ratio is one of the most important aspects when designing the aerodynamic performance of wind turbine blades. These values were determined at $\operatorname{Re}=4 \cdot 10^{4}$ in the smooth flow conditions by using the XFOIL software developed by Drela [12]. The study performance of airfoil by numerical calculation showed that the prototype airfoil of a wind turbine had weaker performance in lower Reynolds number than the thinner profile. The optimal airfoil proved to be the thinnest airfoil AG24, where $C_{l} / C_{d}=45$ at the flow incidence angle of 6 , which is the optimal flow incidence angle for the pitched blades. At low Re, it was not possible to achieve that large $C_{l} / C_{d}$ as that was the case at high Re. In the full-scale conditions, $C_{l} / C_{d}$ can be larger than 150 , which is one of the main reason why small-scale wind turbines cannot be so effective as the full-scale wind turbines.

\subsection{Wind tunnel measurements}

Laboratory experiments were carried out in the Climatic Wind Tunnel (CWT) of the Institute of Theoretical and Applied Mechanics, Czech Academy of Sciences. CWT is designed as a closed-loop boundary layer wind tunnel with controlled flow velocity and temperature. It consists of the climatic and aerodynamic sections. The aerodynamic section provides appropriate conditions to study wind effects on downscaled models of prototype objects.

The wind turbine model was placed on a 6-axis force balance sensor ATI MINI 40, which is able to concurrently measure three forces and three bending moments in the foundation of the structural model. The incoming flow was smooth and uniform with low turbulence intensity $I_{u}=1-2 \%$. The flow speed was measured in front of the wind turbine model rotor in the nacelle model height by using the Prandtl-Pitot tube connected to the Scanivalve pressure system for average pressure measurements. Fig. 2 a shows the wind 
turbine model placed in the aerodynamic wind tunnel test section together with the PrandtltPitot tube.
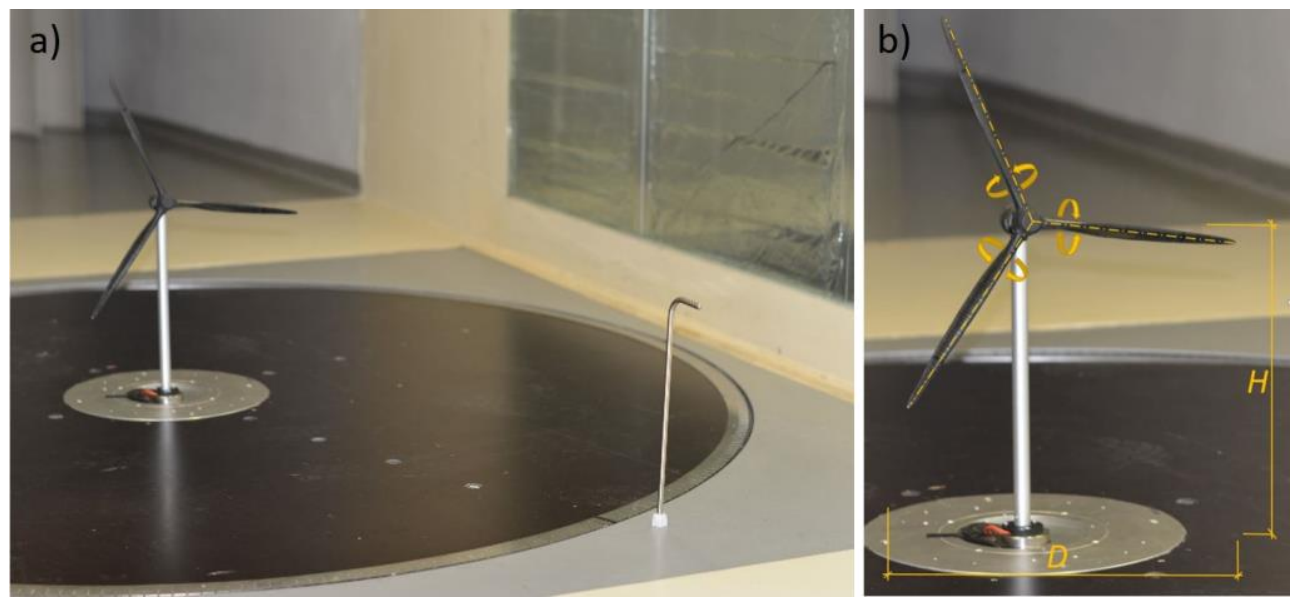

Fig. 2. Model placed on the force balance sensor (a) and characteristic size of wind turbine (b).

Only several previous studies have attempted to directly measure the shaft torque of small-scale miniature turbines due to the difficulties concerning the low shaft torque and space limitations. [13] measured the torque by mounting the generator inside a cylindrical housing with internal ball bearings, where the rotation of the generator was prevented by the deflection of a strain-gauge sensor. In [14], a similar concept was used to measure the mechanical torque. Instead of using a strain-gauge sensor, they measured the torque by adding known weights to the customized moment arm attached to the generator. In spite of the merit of these studies, the calibration procedure of these methods is rather cumbersome and they cannot be easily applied to different operating conditions.

In the present study, the rotation speed was controlled by a three-phase alternator, which allows setting rotational damping and rotation speed. Increasing of damping causes an increase in the electricity gained from the alternator, which makes possible to calculate the power coefficient and the torque coefficient. Converting the electrical power to mechanical power on the shaft is enabled by the properties of the alternator in the entire working range of the alternator.

\section{Results}

Aerodynamic performance of three blade types was investigated at various incoming freestream flow velocities. Fig. 3 shows an almost linear behaviour of the blade rotation velocity when increasing the incoming flow velocity. The fastest blades were detected for the airfoil type AG24. This means that this blade can be considered as the most effective one. The linearity of the graphs also means that in the entire Reynolds number regime $\mathrm{Re}=$ $1 \cdot 10^{3}$ to $4 \cdot 10^{4}$ no sharp transition regimes (similar e.g. to the crisis at cylinders) are present. The maximum velocity of tip speed of experimental model blades reached up to $288 \mathrm{~km} / \mathrm{h}$, i.e. the Mach number of the tip was $M=0.23$. This value is very close to the air compressibility. Based on this analysis, it was decided, to use the AG24 blades in subsequent measurement. 


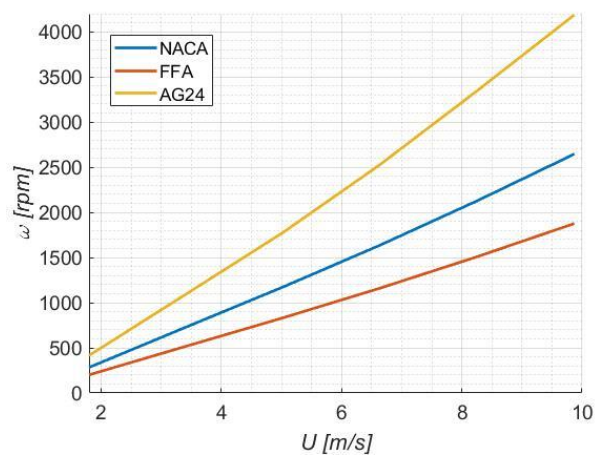

Fig. 3. Speed of spinning with various incoming freestream flow velocities.

The relevant results from the point of view of the wind turbine design are the aerodynamic coefficients. They are dimensionless values determined by the following equations

$$
\begin{gathered}
C q=\frac{2 M_{x}}{U^{2} \rho_{\text {air }} \pi R^{2}} \\
C t=\frac{2 F_{x}}{U^{2} \rho_{\text {air }} \pi R^{2}} \\
C p=\frac{2 P}{U^{3} \rho_{\text {air }} \pi R^{2}}
\end{gathered}
$$

$C p$ is the coefficient of the mechanical power on the wind turbine model shaft, $C q$ is the torque coefficient on the wind turbine model shaft, $C t$ is the thrust coefficient, $M_{x}$ is the torsion moment on the wind turbine model shaft, $P$ is the mechanical power, $F_{x}$ is the drag force coefficient, $U$ is the flow speed and $\rho_{\text {air }}$ is the air density and $r$ is variable distance from the rotor centre to the top of the blade.

a)

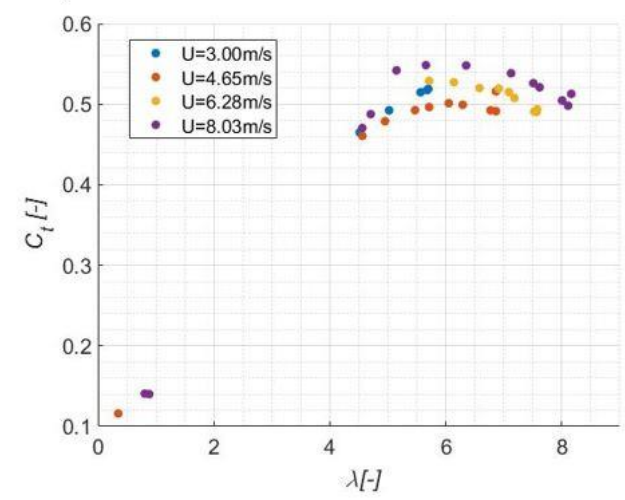

b)

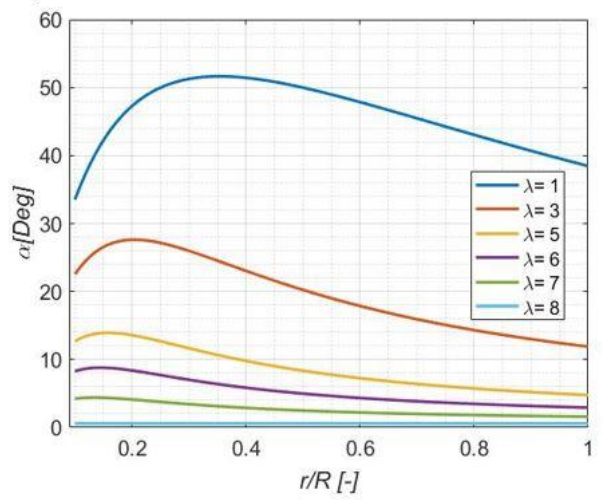

Fig. 4. Aerodynamic a) thrust coefficient and b) flow incidence angle. 
Fig. 4a shows the thrust coefficient in the relation to the tip-speed ratio at four different freestream flow velocities. The thrust force coefficient $C_{t}$ for the low tip-speed ratio is significantly lower than in the conditions with the high tip-speed ratio. This phenomenon is caused by the large flow incidence angle acting on the airfoil while the wind turbine model is in the stall regime, which generated low thurst forces. Fig. 4b depicts the calculated flow incidence angle against the nondimensionalized blade radius at various tip-speed ratios. For the tip-speed ratio between 1 and 3, the flow incidence angle is quite large and the airfoil is in the stall, see also the diagram in Fig. 1b.
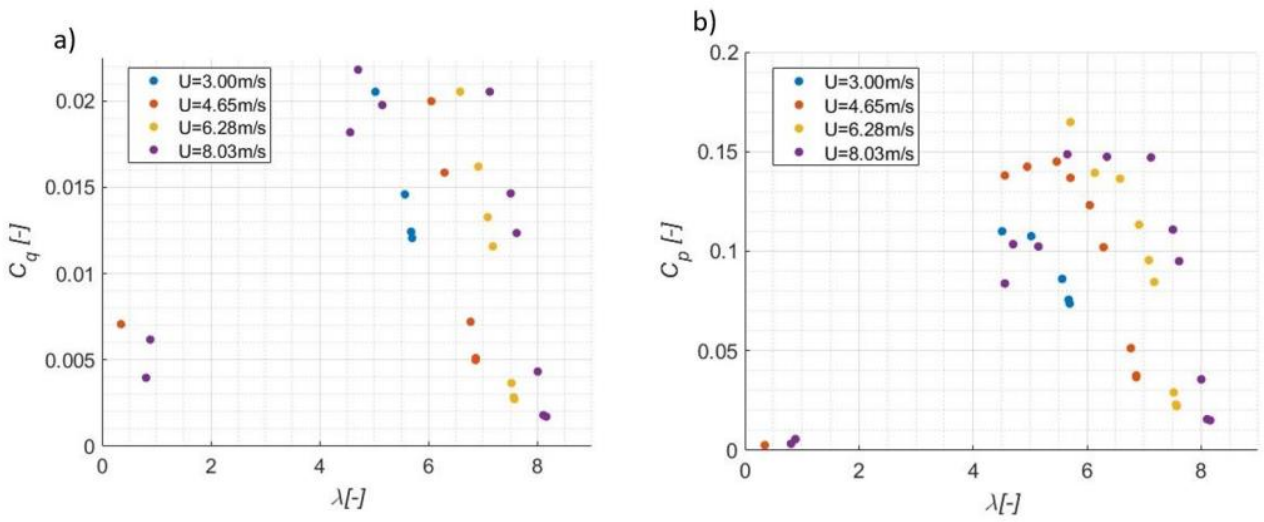

Fig. 5. Aerodynamic a) torque coefficients and b) power coefficient.

Fig. 5a shows the shaft torque coefficient as a function of the tip-speed ratio. It increases with decreasing the tip-speed ratio, which corresponds well with the results in [15]. Only three of all the measured cases, related to the flow speed $8 \mathrm{~m} / \mathrm{s}$ in the interval of $\lambda=4.4-5.2$, are not in the same trend as another measurement. This is because the tipspeed ratio is on the boundary for the stall regime to appear, while simultaneously a part of the blades is in the stall regime causing a decrease of the torque.

The power coefficient is most sensitive to different flow velocities and consequently to Re. The diagram in Fig. 5b shows that the higher flow velocity is the most effective mean so the wind turbine model can achieve the highest tip-speed ratio. The maximum reached tip-speed ratio from all of the presented measurement was $\lambda=8$. This is given by design of presented blades, primarily the twist of the blades and the blade pitch angle. Fig. 4b confirms the result of the measurement as the flow incidence angle at $\lambda=8$ is almost zero so that the blades move fast but are not capable of generating the torque moment. Power coefficient is $C p=0.15$. Although it is approximately three times lower than the most efficient real wind turbine, one has to be aware that the small-scale wind turbine model in the flow conditions at low Re cannot reach higher efficiency, unless tested at very high Re.

\section{Conclusions}

The present study is an attempt to manufacture the miniature small-scale wind turbine model to achieve the realistic values of the thrust force and power coefficient. A small-scale wind turbine model designed for this purpose achieves theoretical values of the thrust force. The aerodynamic power and torque correspond to the theoretical analysis and available references [15] for the selected ratio $\mathrm{Cl} / \mathrm{Cd}$. The maximal efficiency and torque force correspond with the design intention of an ideal tip-speed ratio $\lambda=6$. The unique and 
efficient system of the control of the blade speed with the simultaneous measurement of the shaft torque as well as the mechanical power was designed. The presented results increase the knowledge of the small-scale wind turbine behaviour subjected to the uniform flow and are useful for further numerical analyses and experiments. They may also serve for the analysis of the small-scale wind turbine power control in complex terrain and turbulent flow.

The authors acknowledge the Croatian Science Foundation IP-2016-06-2017 (WESLO) support.

\section{References}

1. P. Fuglsang, H.A. Madsen, Optimization method for wind turbine rotors, Journal of Wind Engineering and Industrial Aerodynamics, 80(1-2), 191-206 (1999)

2. H. Kozmar, D. Allori, G. Bartoli, C. Borri Complex terrain effects on wake characteristics of a parked wind turbine, Engineering Structures, 110, 363-374 (2016)

3. Y. Yang, M. Gu, S. Chen, X. Jin, New inflow boundary conditions for modelling the neutral equilibrium atmospheric boundary layer in computational wind engineering, Journal of Wind Engineering and Industrial Aerodynamics, 97(2), 88-95 (2009)

4. K. Howard, L. Chamorro, M. Guala, An Experimental Case Study of Complex Topographic and Atmospheric Influences on Wind Turbine Performance, Proceedings of the 51st AIAA (2013)

5. S. Sunada, A. Sakaguchi, K. Kawachi, Airfoil section characteristics at a low Reynolds number. Journal of Fluids Engineering, 119, 129-135 (1997)

6. E. Laitone,Wind tunnel tests of wings at Reynolds numbers below 70 000, Experiments in Fluids, 23, 405-409 (1997)

7. S. Sunada, T. Yasuda, K. Yasuda, K. Kawachi, Comparison of wing characteristics at an ultra low Reynolds number, Journal of Aircraft, 39 , 331-338 (2002)

8. A. Pelletier, T.J. Mueller, Low reynolds number aerodynamics of low-aspect-ratio, thin/flat/cambered-plate wings, Journal of Aircraft, 37, 825-832 (2000)

9. T.Y. Chen, L.R. Liou, Blockage corrections in wind tunnel tests of small horizontal-axis wind turbines, Experimental Thermal and Fluid Science, 35, 565-569 (2011)

10. H. Glauert, The elements of airfoil and airscrew theory (Cambridge University Press, 1926)

11. DNV-OS-J101, Design of Offshore Wind Turbine Structures. Det Norske Veritas (2014).

12. M. Drela, XFOIL 6.94 User Guide, Massachusets istittute of Technology Cambridge, Massachusets, USA

13. H.S. Kang, C. Meneveau, Direct mechanical torque sensor for model wind turbines, Measurement Science and Technology, 21 (2010)

14. K. Howard, J. Hu, L. Chamorro, M. Guala, Characterizing the response of a wind turbine model under complex inflow conditions, Wind Energy, 18, 729-743 (2014)

15. H. Jiang, Z. Chen, Y. Zhao, Torque Limit of Horizontal Axis Wind Turbine, Proceedings of 2012 International Conference on Mechanical Engineering and Material Science, 148-155 (2012) 